\title{
Impact Analysis of Roller System Stability for Four-High Mill Horizontal Vibration
}

\author{
Xiao-bin Fan, ${ }^{1}$ Yong Zang, ${ }^{2}$ Yuan-kui Sun, ${ }^{1}$ and Ping-an Wang ${ }^{1}$ \\ ${ }^{1}$ School of Mechanical and Power Engineering, Henan Polytechnic University, Jiaozuo 454003, China \\ ${ }^{2}$ Mechanical Engineering Institute, University of Science and Technology Beijing, Beijing 100083, China
}

Correspondence should be addressed to Xiao-bin Fan; fanxiaobin@hpu.edu.cn

Received 26 March 2016; Revised 18 June 2016; Accepted 26 June 2016

Academic Editor: Chao Tao

Copyright (c) 2016 Xiao-bin Fan et al. This is an open access article distributed under the Creative Commons Attribution License, which permits unrestricted use, distribution, and reproduction in any medium, provided the original work is properly cited.

In order to study the hot Compact Strip Production (CSP), four-high mill vibration characteristics, and vibration suppression method, the roller system structure stability was analyzed and calculated at first in the paper. And then, the mill stand gap was measured at field and its influence on roll transverse vibration was analyzed. The drum gear coupling effect on the roller system stability and the automatic balance conditions of the coupling transmission torque were studied; the influence of axial force caused by the roller cross on the system stability was analyzed. Finally, the roller transverse friction chatter vibration mechanics model was established; the simulation analysis was carried out with eliminating mill house-bearing clearance and adding floating support for coupling, respectively. And the characteristics of the roller "jump vibration" were studied. We applied copper gaskets to eliminate or reduce mill house-bearing clearance for suppressing the rolling mill vibration on the spot; the test results show that the roller transverse vibration was suppressed after eliminating clearance.

\section{Introduction}

For the convenience of installation work roller, there is clearance between work roller bearing seat and mill house in fourhigh mill, which makes work roller in an unstable state when there is no fixed side force on it. So it should have an offset $e$ between work roller axis center and backup roller, and its value certainty principle is to make the lateral reaction force of the frame acting on work roller bearing always greater than zero, and the force direction is constant. Modern four-high mills all set corresponding offset value $[1,2]$, such as 2050 CVC four-high rolling mill and 4300-medium plate mill of the German SMS, four-roll rolling mill of the British DAVY, 1580-pair cross four-high rolling mill of Japan Mitsubishi heavy industries. But these modern four-high mills with offset all have roller parallel positioning instability, and with the increasing of rolling load and speed, their disadvantages will become more serious. For these CSP mills, the offset value is $1.0 \sim 1.2 \mathrm{~mm}$, which also facilitates replacement roller installation and disassembly and serves as a compensation of bearing thermal expansion space.
Although scholars have conducted a lot of research on rolling mill vibration, because the problem is too complicated, it has not been solved well. A. Bar and O. Bar presented the numerical analysis of vibrations with moderate frequencies excited during the milling process taking into account the transportation motion of the strip and its inertial properties [3]. Bar and Świątoniowski built the nonlinear mathematical model of oscillated system: the continuous group of the rolling stands coupled by transferring strip [4]. Panjković et al. found that the frictional conditions in the roll gap are the principal cause of chatter in this mill and the frictional conditions appear to be associated with the thickness and properties of oxide formed on rolls [5]. Furumoto et al. designed a chamber in mill stabilizing device and optimized its size [6]. Kim et al. modeled a rolling mill that includes the driving system by multibody dynamics to investigate the cause and characteristics of the chatter vibration. The chatter frequency was $1190 \mathrm{~Hz}$ and was caused by the rolling force. The amplitude of chatter vibration could be reduced by controlling the speed of the roll and the static and dynamic 


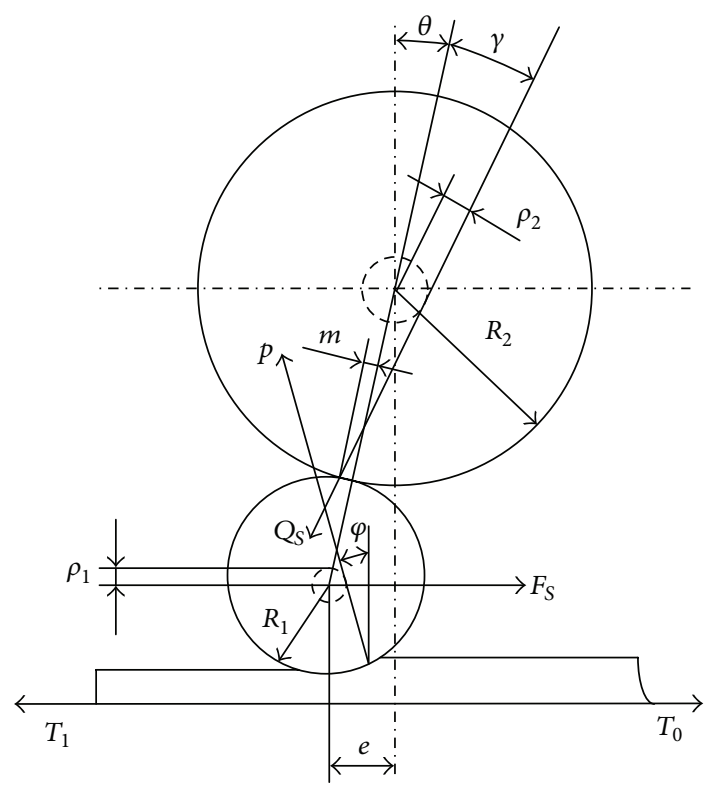

FIGURE 1: Upper roll system force diagram.

components of the rolling force [7]. They also proposed a mathematical model of a cold rolling mill including the driving system and a novel combination of the direct integration method and quasistatic analysis to solve the model efficiently and found the horizontal chatter vibration had a strong effect on the dynamic characteristics [8]. Swiatoniowski and Gregorczyk presented a probabilistic model of the friction phenomena on the work-backup rolls contact surface and fount that such character of the disturbance in distribution of zones with static and kinetic friction can be regarded as one of the sources of self-excited vibrations appearing in the system consisting of a rolling mill and a strip [9]. Amer et al. studied the torsional vibration reduction for rolling mill's main drive system via negative velocity feedback under parametric excitation and found the resonance condition is the first natural frequency vibration as one of the worst resonance cases [10].

Usually, rolling mill vibration research does not distinguish whether rolling mill roll system is stable, and mechanics model is set up setting the mill under stabilization. A single vibration as the coupling mode with roll vertical, horizontal, axial, torsional, cross, and swing space vibration behavior is analyzed. Actually, roller vibration will damage roll system stability and cause bearing horizontal or vertical vibration. Therefore, the CSP four-high mill vibration characteristics were studied from the mill roll system stability and lateral dynamic behavior of the roll system in this paper.

\section{Roller System Structure Stability Calculation}

Work roller force analysis is shown in Figure 1, where $Q_{s}$ is the force on work roller by backup roller, $P$ is the rolling force, $F_{s}$ is the work roll bearing reaction force by mill house, $m$ is the friction arm distance $(0.2 \mathrm{~mm})$ between work roller and backup roller, $T_{1}$ and $T_{0}$ are the front and back tension, $R_{1}$ and $R_{2}$ are the work roll and backup roll radius, $\rho_{1}$ and $\rho_{2}$ are work roll and backup roll bearing friction circle radius, $\theta$ is the included angle between the work roll and backup roll defiled line, and there is $\sin \theta=e /\left(R_{1}+R_{2}\right) ; \varphi$ is deviation angle of front and back tension impact on rolling force direction, and there has been $\sin \varphi=\left(T_{1}-T_{0}\right) / 2 P ; \gamma$ is the included angle between roller defiled line and reaction force $Q_{s}$, and there has been

$$
\sin \gamma=\frac{\left(\rho_{2}+m\right)}{R_{2}} .
$$

Due to the included angle between the roller and drum gear spindle axis, the spindle will produce additional moment $M_{H}$ on the roller; namely, in the horizontal direction, it will make the roller produces additional horizontal force $F_{H}$ and $F_{H}=M_{H} / L$. The force on the side is pointing in the direction of work roll shift direction and the force is departure from the direction of work roll shift on the other side and may cause two rollers' crossing, where $L$ is the distance between the center lines of the both work rollers bearing seat and $M_{H}=M_{A} \cdot \tan \alpha, \alpha$ is included angle between the spindle and roller and $M_{A}$ is the sum of the rolling torque $M_{R}+$ support roller rotation torque $M_{B}+$ work roller bearing friction consumption torque $M_{W}$; namely,

$$
M_{A}=P \cdot a+Q_{S} \cdot c+F_{S} \cdot \rho_{1},
$$

where $a$ is rolling force arm. When $T_{1}>T_{0}, a=R_{1} \cdot \sin (\beta-\varphi)$, $\beta$ is the roller center angle corresponding to rolling force point without considering tension, and $\beta \approx(1 / 2) \sqrt{\Delta h / R^{\prime}}(1-$ $\left.(1 / 2 \mu) \sqrt{\Delta h / R^{\prime}}\right)$, where $\Delta h$ is reduction and $R^{\prime}$ is the roll flattening radius. The force of backup roller acting on work roller is $Q_{S}=P \cdot \cos \varphi / \cos (\theta+\gamma) \cdot c$ is the arm of force of $Q_{S}$ acting on work roller and $c=m \cos \gamma+R_{1} \sin \gamma$. When $T_{1}>T_{0}, F_{S}=Q_{S} \sin (\theta+\gamma)+P \sin \varphi$. At this time, the work roller system stability condition is that the reaction force of mill house acting on work roller meets $F_{s}^{\prime}>0$ or $F_{S}^{\prime}=F_{S}+F_{H}>0$; namely,

$$
\begin{aligned}
F_{S}^{\prime}= & F_{S}+F_{H} \\
= & P \cos \varphi[\tan \varphi+\tan (\theta+\gamma)] \\
& +P \tan \alpha \frac{\left\{R_{1} \sin (\beta-\varphi)+(\cos \varphi / \cos (\theta+\gamma)) \cdot\left(m \cos \gamma+R_{1} \sin \gamma\right)+\cos \varphi[\tan \varphi+\tan (\theta+\gamma)] \rho_{1}\right\}}{L} .
\end{aligned}
$$




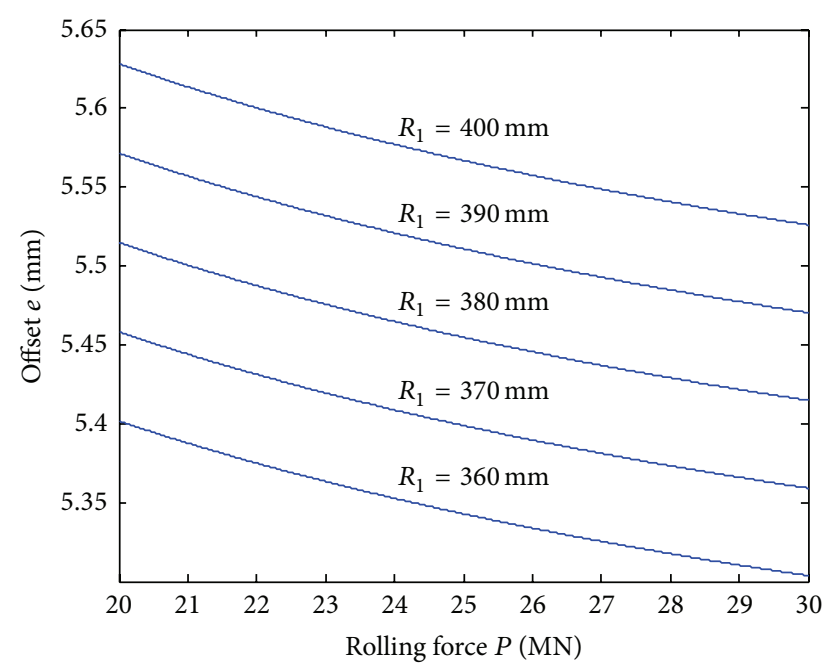

Figure 2: Minimum offset calculation.

If $\cos \varphi \approx 1, \cos (\theta+\gamma) \approx 1$, and $\tan (\theta+\gamma) \approx \sin \theta+\sin \gamma=$ $e /\left(R_{1}+R_{2}\right)+\left(\rho_{2}+m\right) / R_{2}, \tan \varphi \approx \sin \varphi=\left(T_{1}-T_{0}\right) / 2 P$, and then (3) can become as follows:

$$
\begin{aligned}
& \frac{T_{1}-T_{0}}{2 P}+\frac{e}{R_{1}+R_{2}}+\frac{m+\rho_{2}}{R_{2}} \\
& \quad+\frac{2 \tan \alpha}{L}\left[R_{1} \sin (\beta-\varphi)+m \cos \gamma+R_{1} \sin \gamma\right]
\end{aligned}
$$

$>0$.

At this time, the work roller offset should be satisfied as follows:

$$
\begin{aligned}
e> & e_{0}=\left(R_{1}+R_{2}\right)\left\{\frac{T_{1}-T_{0}}{2 P}+\frac{m+\rho_{2}}{R_{2}}\right. \\
& \left.+\frac{2 \tan \alpha}{L}\left[R_{1} \sin (\beta-\varphi)+m \cos \gamma+R_{1} \sin \gamma\right]\right\} .
\end{aligned}
$$

By (5), if one considers the included angle between the spindle and the roller influence, the work roller offset should be increased $(2 \tan \alpha / L)\left(R_{1}+R_{2}\right)\left[R_{1} \sin (\beta-\varphi)+m \cos \gamma+\right.$ $R_{1} \sin \gamma$ ] based on the original.

Minimum offset change scope with the rolling force change under work roller diameter within a certain range is shown in Figure 2. According to the experience in the production, the offset values are usually larger than the theoretical calculation $(3 \sim 5 \mathrm{~mm})$. The offset $e$ of $F 3$ rolling mill is $10 \mathrm{~mm}$; when the work roller diameter is small, it probably meets the requirements of the static stability.

\section{Mill House Clearance and Drum Gear Coupling Effect on the Roll System Stability}

The CSP mill house and bearing plate never change from production in recent 4 years; their wear and tear may be large. So the F3 mill upper and lower work roller bearing seat width of drive side and operation side were measured. In order to improve the measurement accuracy, 4 points of bearing plate

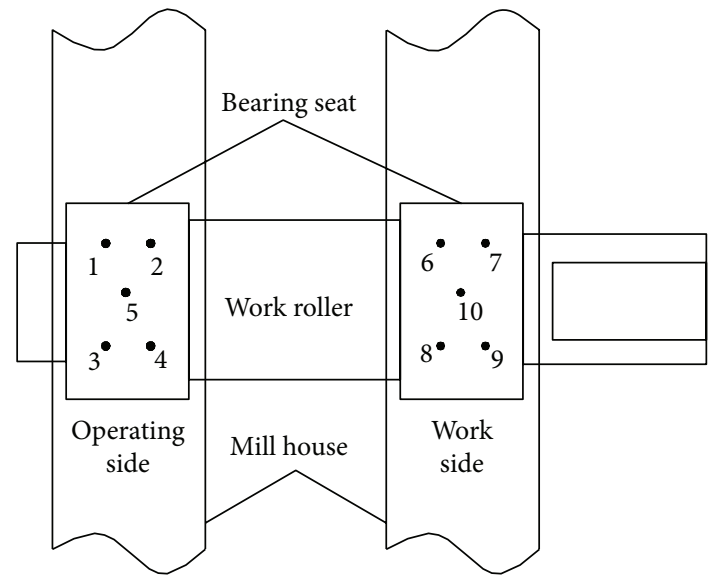

FIGURE 3: Clearance measurement.

were selected and shown in Figure 3 (1 4 points on operating side, 6 9 points on drive side), and then the average was got. Five points were selected on mill house plate ( $1 \sim 5$ points on operation side, $6 \sim 10$ points on drive side), and then the average was got. The measurement results of the bearing seat and mill house and the original design values are shown in Table 1 .

From Table 1, bearing width has negative deviation; the negative deviation is around $0.5 \mathrm{~mm}$. Mill house width has positive deviation; the deviation value is around $1.8 \mathrm{~mm}$. The upper work roller clearance averages of drive side and operation side are $2.343 \mathrm{~mm}$ and $2.442 \mathrm{~mm}$, respectively. The lower work roller clearance averages of drive side and operation side are $2.194 \mathrm{~mm}$ and $2.319 \mathrm{~mm}$, respectively. Obviously, the F3 mill house and bearing seat clearance are much larger original design requirement (transition fit); namely, the gap provides necessary conditions to roller transverse vibration.

According to the mechanical transmission theory, the condition of spindle can be normally operated as follows: gear shaft axis, roller axis, and drum gear spindle axis must be located on the same plane, and we must ensure that both the included angles with the spindle axis are equal. But with roll bearing seat and mill house plate wear (in the outlet side mainly), roll connected end to the spindle will shift toward the exit side. Then, the spindle will produce angle between the vertical planes; namely, the spindle axis, gear axis, and roller axis are not in a plane. This will make the spindle have additional torque on the roller, and the additional torque will make the work roller produce deflection movement in the horizontal direction, which will make the bearing seat acting force uneven (as shown in Figure 4) and the bearing seat tilt (Figure 5) and roller axis cross (Figure 6) and other adverse condition. The additional horizontal force directions of the operation side and transmission side of the roller are opposite, under the combined action of the clearance and the additional bending moment, which will make the roller bearing seat have crash tendency on mill house (this is confirmed from the scene that bearing seat entrance side plate also has certain wear and tear). In addition, because of the bearing seat plate force and uneven wear (Figure 4), it 
TABLE 1: Mill house and bearing seat clearance design value and measurements (unit: $\mathrm{mm}$ ).

\begin{tabular}{lcccc}
\hline & \multicolumn{2}{c}{ Bearing seat width } & \multicolumn{2}{c}{ Mill house width } \\
& Drive side & Operating side & Drive side & $890_{-0.16}^{0}$ \\
\hline $\begin{array}{l}\text { Design size } \\
\text { Measurement }\end{array}$ & $890_{-0.16}^{0}$ & $900_{-0.16}^{0}$ & & \\
$\quad$ Upper roller & $890-0.490$ & $900-0.540$ & $890+1.853$ & $900_{-0.16}^{0}$ \\
$\quad$ Lower roller & $890-0.445$ & $900-0.495$ & $890+1.749$ & $900+1.902$ \\
\hline
\end{tabular}
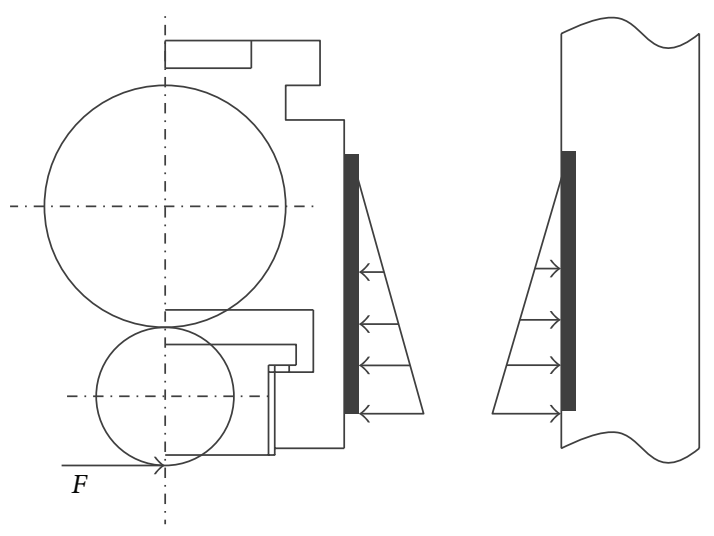

FIGURE 4: Bearing seat and mill house force diagram.

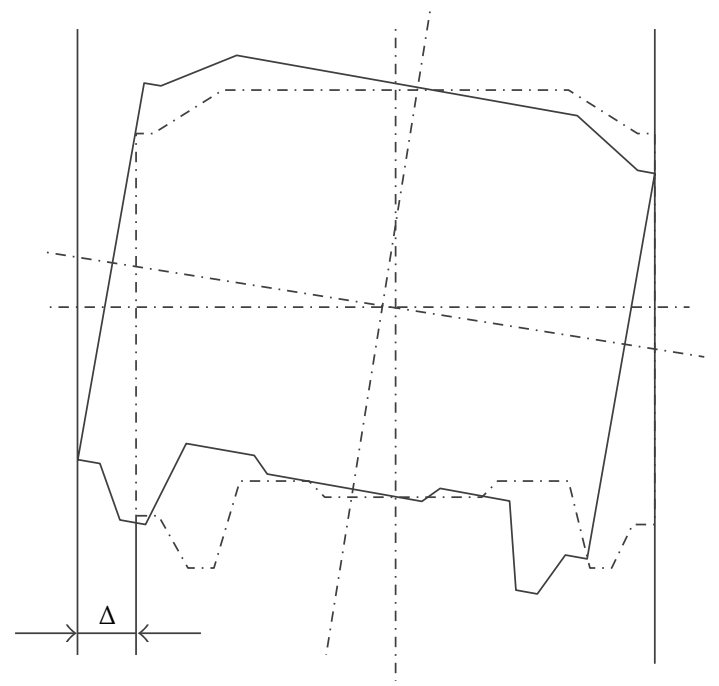

Figure 5: Bearing seat tilt.

will destroy the roller system stability under roller insufficient constraints.

Work roller axial force caused by backup roller cross and rolled strip cross is divided into roller axial force and deformation zone axial force. And the deformation zone axial force is dominant, which mainly comes from axial component of the friction force, and its direction depends on the metal particle longitudinal and transverse sliding velocity direction relative to the roller surface; its value is related to rolling pressure and the friction condition, as follows:

$$
F_{a}=\int_{-b / 2}^{b / 2} \int_{0}^{l} \frac{\tau_{A}}{\cos \theta} d x d y
$$

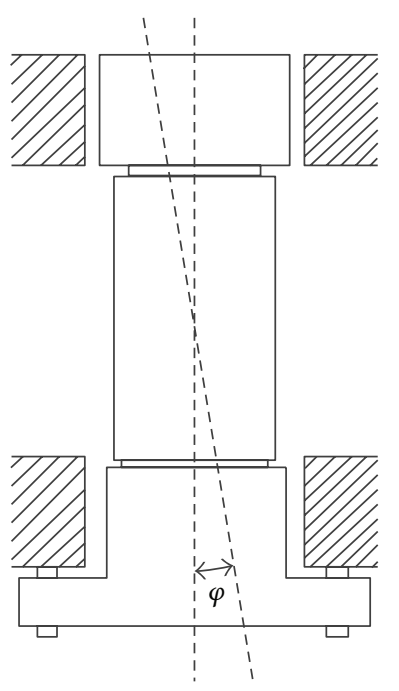

Figure 6: Roller cross.

where $b$ is the rolled strip width, $l$ is the deformation zone length, $\theta$ is the contact angle and $\tan \theta=-(1 / 2) h_{x}^{\prime}=$ $\left(\left(h_{0}-h_{1}\right) / l\right)(1-x / l), h_{x}$ is rolled strip thickness at the $x$ coordinate deformation zone, and $h_{0}$ and $h_{1}$ are the rolled strip thickness at entrance and export. $\tau_{A}$ is axis component of deformation area friction force. According to the axial force vector diagram in Figure 7, there has been $\tau_{A}=\tau|\sin (\psi+\varphi)|$, where $\tau$ is the deformation area friction, $\psi$ is sliding angle between metal and the rollers, $\varphi$ is roller crossing angle, $v$ is the arbitrary point metal flow rate in deformation area, and $v_{R}$ is rotational speed of roller surface. Thus, according to the $F_{a}$ calculation equation, the relationship curves between axial force and reduction rate and bearing-mill house clearance are shown in Figure 8. It can be seen that the axial force caused by roller cross increases with reduction rate and clearance increase; its effect on the system cannot be ignored.

The F3 mill upper work roller vibration acceleration testing time-domain parameters on field is shown in Table 2. It can be seen that although axial force is less than $1 \%$ of the rolling force, roller axial vibration acceleration amplitude is close to vertical acceleration and lateral acceleration, so the axial force (caused by rollers cross) destruction on the system stability cannot be ignored; we should try to eliminate the gap between roller bearing seat and the mill house; at the same time, it also shows the gap has important influence on roller vibration.

In addition, because the length of mill drum gear coupling is about $5 \mathrm{~m}$ and its weight is about 7 tons and it has no 


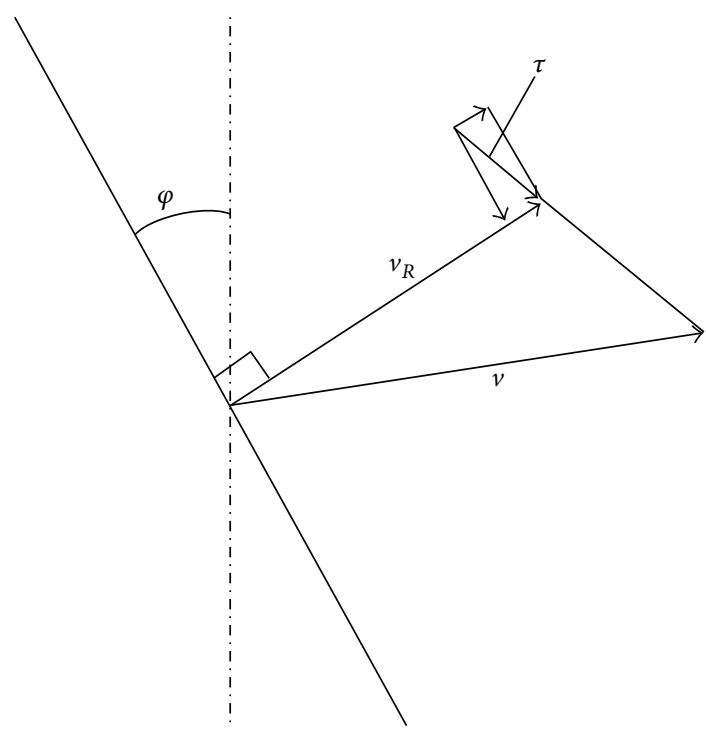

Figure 7: Axial force vector diagram.

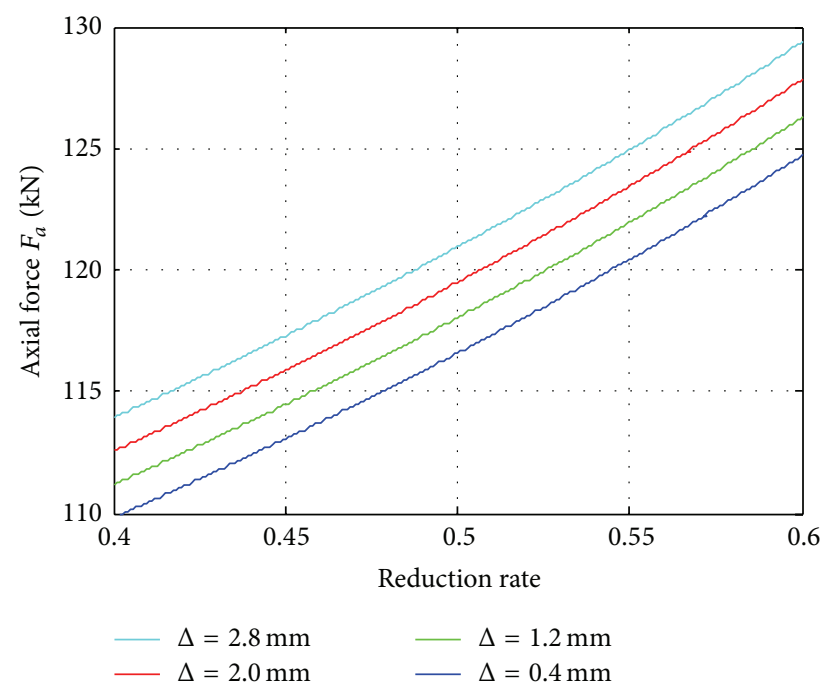

FIgURe 8: Axial force theory diagram.

TABLE 2: Roller vibration measurement (unit: $\mathrm{m} \cdot \mathrm{s}^{-2}$ ).

\begin{tabular}{lccc}
\hline & Peak value & $\begin{array}{c}\text { Average } \\
\text { amplitude }\end{array}$ & $\begin{array}{c}\text { Effective } \\
\text { value }\end{array}$ \\
\hline Vertical acceleration & 1.6383 & 0.4096 & 0.5074 \\
Lateral acceleration & 2.1308 & 0.4341 & 0.5499 \\
Axial acceleration & 1.2711 & 0.2919 & 0.3599 \\
\hline
\end{tabular}

support, in the presence of the spindle imbalance, due to the internal or external gear eccentricity and the gravity center offset, these will bring large unbalanced force and affect the roll system dynamic stability. In the presence of drum gear tooth tip clearance, centrifugal force $F_{c}$ will be generated:

$$
F_{c}=\frac{m \Delta y n^{2}}{1.82 \times 10^{5}} \mathrm{~N},
$$

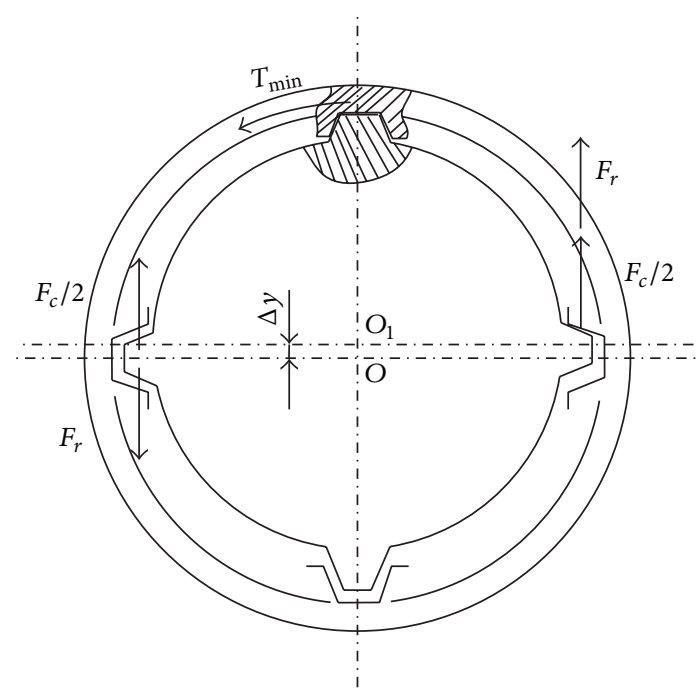

FIGURE 9: Gear coupling centrifugal force.

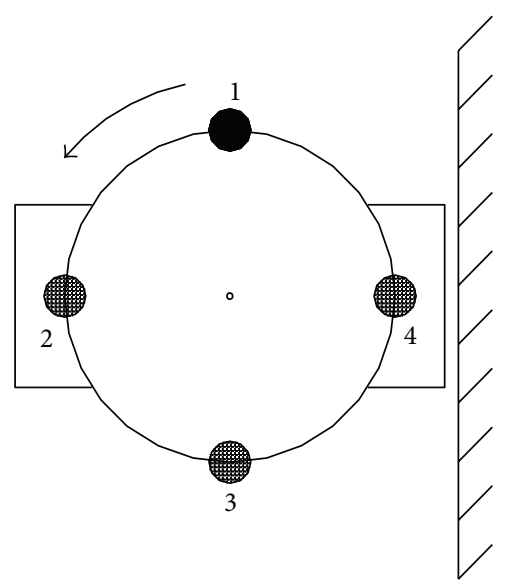

FIGURE 10: Unbalanced force diagram.

where $m$ is the outer gear shaft spindle weight, $\mathrm{kg} ; n$ is the coupling working speed, $\mathrm{r} / \mathrm{min} ; \Delta y$ is the relative radial displacement, mm, as shown in Figure 9. As a result of the existence of the centrifugal force, in order to make the interior and exterior of tooth coupling automatically, work roller needs to transmit the minimum torque $T_{\min }$; namely,

$$
T_{\min }=\frac{\mathrm{men}^{2} d}{3.64 \times 10^{5}}(\mathrm{~N} \cdot \mathrm{mm}),
$$

where $m$ is the weight of the intermediate shaft or internal gear ring, $\mathrm{kg} ; e$ is inside and outside of the teeth relative eccentricity, $\mathrm{mm} ; n$ is the coupling speed, $\mathrm{r} / \mathrm{min} . d$ is tooth reference circle diameter, $\mathrm{mm}$. Substituting parameters into (8), we get $T_{\min }=4.52 \times 10^{7} \mathrm{~N} \cdot \mathrm{m}$, and the measured torque is $4 \times 10^{5} \mathrm{~N} \cdot \mathrm{m}$; that is, it cannot meet self-centering requirement; there always is centrifugal force or unbalanced force.

The unbalanced force on bearing seat diagram is shown in Figure 10; there, point 1 shows unbalanced force upward, point 2 shows the unbalanced force toward left which makes 


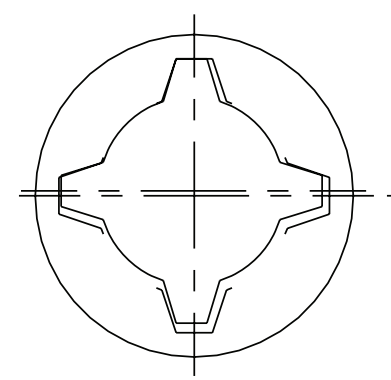

(1)

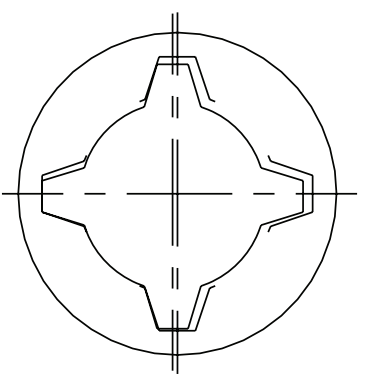

(2)

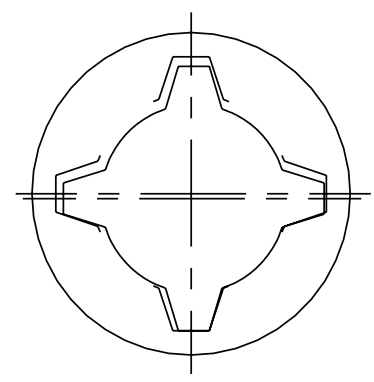

(3)

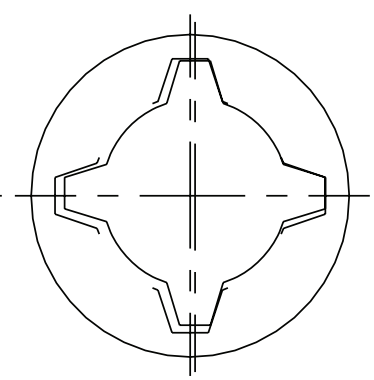

(4)

Figure 11: Drum tooth coupling mesh diagram.

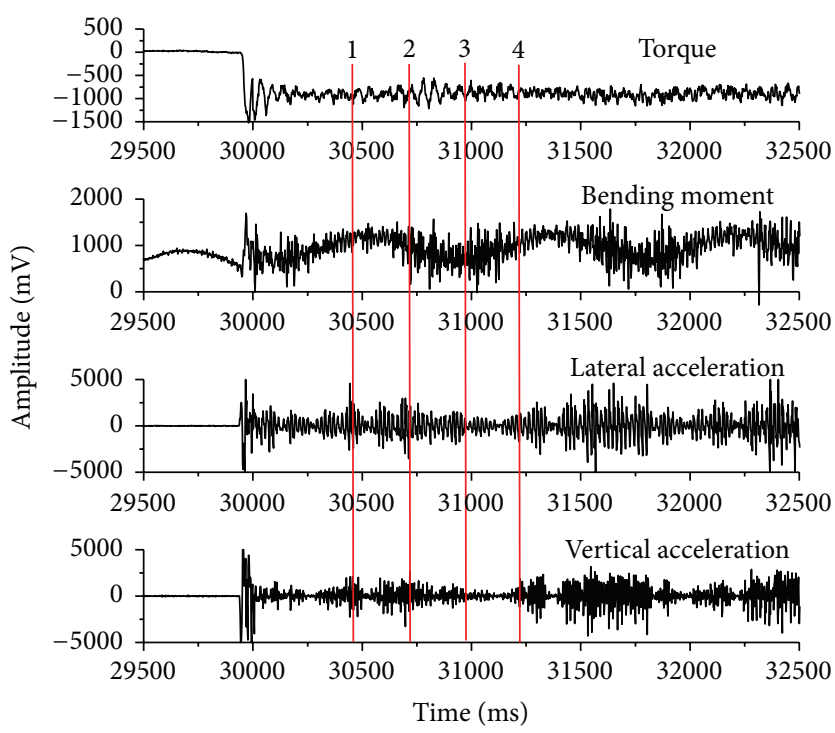

(a)

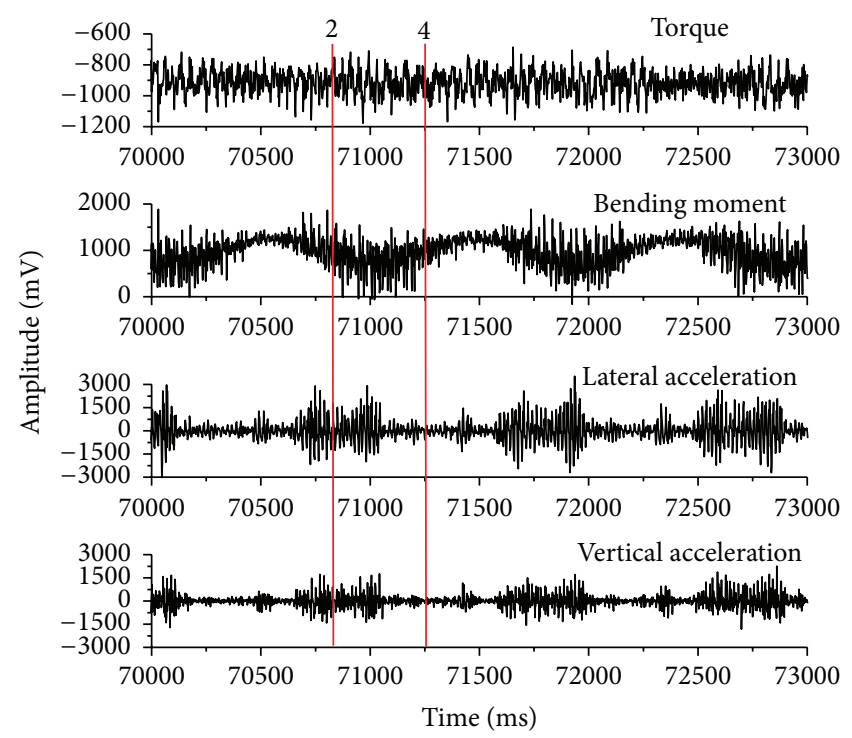

(b)

FIGURE 12: Roller system vibration measurement results.

the bearing seat on left from mill house, point 3 shows unbalanced force downward, and point 4 shows unbalanced force to the right and has a tendency to make the bearing seat pressure on the mill house. For each point corresponds to 1 4, drum gear meshing clearance is shown in Figure 11 accordingly.

Gear spindle shaft torque, bending moment, roller transverse vibration, and vertical vibration test curve are shown in Figure 12. Figure 12(a) shows the strip bite stage and Figure 12(b) shows strip rolling intermediate stage. In the figure, plumb line 1 is corresponding to spindle top point, plumb line 2 is corresponding to spindle left point, plumb line 3 is corresponding to spindle bottom point, and plumb line 4 is corresponding to spindle right point, and they are corresponding to Figures 10 and 11. From Figure 12(a), in the initial stage of strip entrance when drum teeth are in upper and lower vertices, namely, perpendicular lines 1 and 3, meshing impact has greater influence on roller vibration. At stable rolling stage, in the left vertex (at perpendicular line 2), the vibration is most violent, and in the right vertex, the vibration nearly disappeared. This is because unbalanced force makes roller depart from the mill house and be in an unstable state at the left point and makes meshing impact increase sharply. On the contrary, the imbalance force makes roller close to mill house and will not produce unstable state in right vertex. Thus, this makes the waveform produce "gourd" shape; this is also because some mills rarely have this vibration shape equipped with slider or cross type spindle.

In order to determine the vibration nature and find out the vibration source, considering the characteristics of the collective of upper and lower roll drive, speed frequency map values contained the $F 3$ first-order natural frequency of torsional vibration system, the first-order natural frequency of stand vertical vibration system, and the mill stand thirdorder natural frequency of transverse vibration system. Concentrated mass-spring model was adopted for the main drive system, and the first four-order natural frequency is $21.7 \mathrm{~Hz}$, $73 \mathrm{~Hz}, 87.3 \mathrm{~Hz}$, and $229 \mathrm{~Hz}$, respectively. The rolling mill 


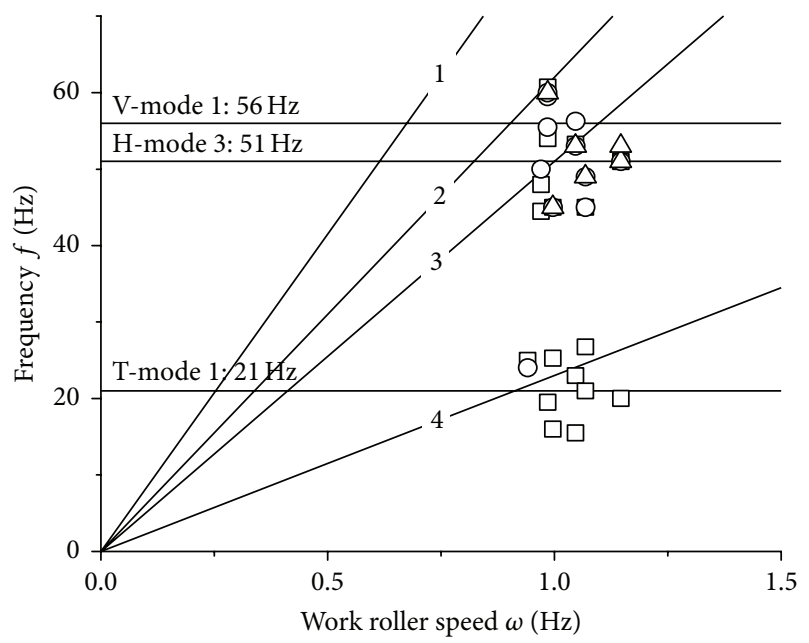

1-4: meshing frequency of middle shaft gear, reducer, arc tooth spindle, and pinion stand

○, $\triangle, \square$ : work roller measured vertical and horizontal acceleration, roll torque dominant frequency

$\mathrm{V}, \mathrm{T}$-mode 1: first natural frequency of vertical and torsional vibration system

H-mode 3: the third natural frequency of horizontal vibration system

FIGURE 13: F3 rolling mill speed map.

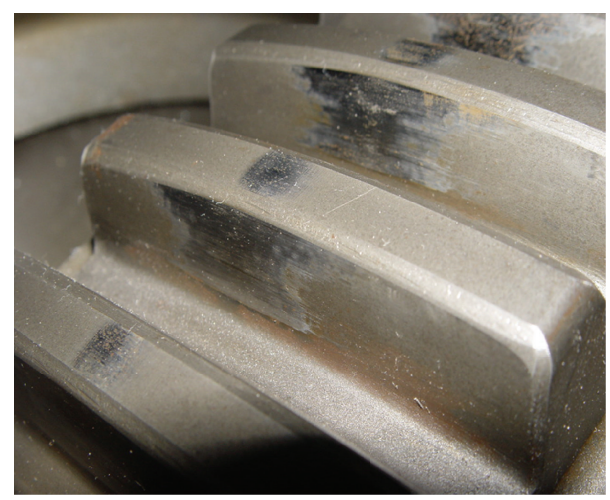

FIGURE 14: Spindle arc tooth wear photos.

transverse vibration natural frequency was calculated with finite element method, and within the roller diameter available range, the first three-order natural frequency of the roll system horizontal vibration is $4.80 \sim 4.99 \mathrm{~Hz}, 50.9 \sim 55.5 \mathrm{~Hz}$, and $55.5 \sim 82.4 \mathrm{~Hz}$, respectively [11].

$$
f(x, \dot{x})= \begin{cases}-\left(k_{1}+k_{2}\right) x+A \dot{x}-B \dot{x}^{3} & x \geq 0 \text { or } x \leq-\Delta_{\max } \\ -k_{1} x+A \dot{x}-B \dot{x}^{3} & -\Delta_{\max }<x<0, \text { contact pressure is zero, }\end{cases}
$$

where $k_{1}$ is the linear stiffness coefficient between work roller and mill house left side, $k_{2}$ is linear stiffness coefficient of mill house pillar, $A$ is the linear damping coefficient, $B$ is the
It can be seen from Figure 13 that the measured vibration main frequency has almost linear relationship with speed, and the $20 \mathrm{~Hz}$ torsional vibration is mainly around concentrated in the gear mesh frequency of distribution box and the first-order natural frequency of torsional vibration system intersection. The $50 \mathrm{~Hz}$ vibration mainly concentrated in the drum gear meshing frequency and the first-order natural frequency of the vertical vibration system intersection. The $F 3$ rolling mill main vibration mode is horizontal and vertical vibration; vibration main frequency is $50 \mathrm{~Hz}$ or so and has approximate linear relationship with the rolling speed. The frequency is consistent with drum gear shaft gear meshing frequency, first-order natural frequency of vertical vibration system, and the-third order natural frequency of horizontal vibration, which indicates that the drum gear meshing impact excites the stand vertical vibration system first-order and the horizontal vibration third-order resonance. It also can be seen from the spot photos (Figure 14) that the spindle drum gear removed from mill has wear and tear; both sides of the drum gear tooth and top all produced excessive wear phenomenon. So it will be beneficial to eliminate vibration mill if drum tooth meshing clearance is eliminated.

\section{Roller Horizontal Friction-Impact Vibration Characteristic Analysis}

Combined with structural characteristics of the roll system, roll horizontal vibration dynamic model is set up. According to the roll loading characteristics, when the extraneous force is more than offset drift force $F_{e}$ and has a gap between roll bearing seat and the mill house, the work roller bearing seat may jump from the mill house, and there is bearing impact on mill house conditions; the system stability is damaged at this time. Considering the mill housing after elasticity, we make it as an elastic collision process in this paper. Based on this, the roller horizontal vibration system "jump" model was set up in Figure 15, and the diagrams (a), (b), and (c) show work roll bearing pressing on the right side of the housing pillar and bearing seat in the middle and bearing pressing on the left side of the housing pillar in sequence. In the figure, $F_{d}$ is the interface friction damping force. So its motion differential equation is as follows:

$$
m \ddot{x}+f(x, \dot{x})=F \cos \Omega t \cos 51 \Omega t,
$$

where $m$ is the weight of work roller, $F$ is extraneous force, $\Omega$ is the roll rotation frequency, and $f(x, \dot{x})$ expression is as follows:

nonlinear damping coefficient, and $\Delta_{\max }$ is the gap between bearing seat and mill house considering the roller crossover and bearing seat tilt. Then, the numerical simulation system 


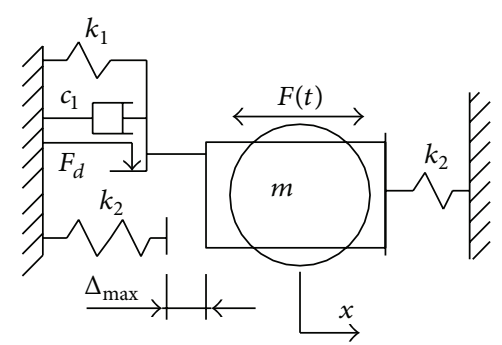

(a)

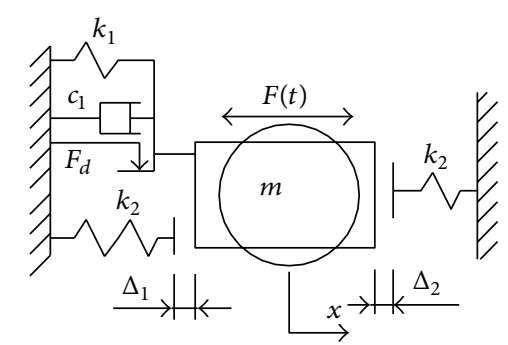

(b)

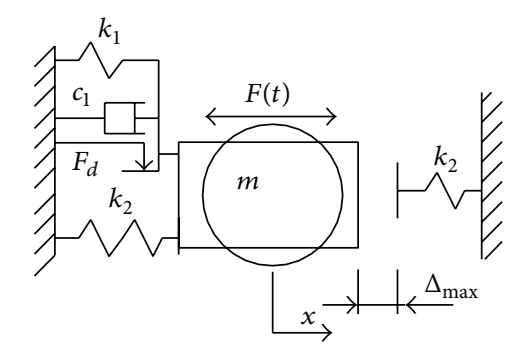

(c)

FIGURE 15: Roller-mill impact vibration mechanics model.

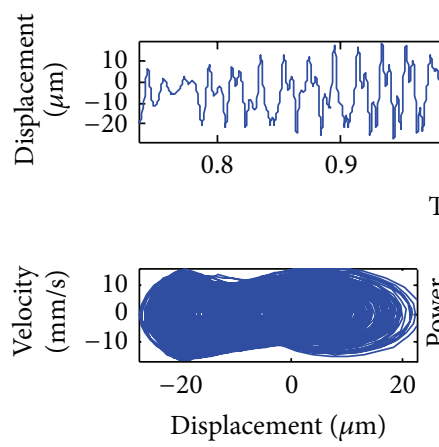

FIGURE 16: F3 horizontal response without measures.

was carried out with the following set of basic parameters or a certain fluctuation: $m=1 \times 10^{5} \mathrm{~kg}, k_{1}=1 \times 10^{10} \mathrm{~N} / \mathrm{m}, k_{2}=$ $1 \times 10^{11} \mathrm{~N} / \mathrm{m}, \Omega=2 \pi, A=4 \times 10^{6} \mathrm{~N} \cdot \mathrm{s} / \mathrm{m}, B=4 \times 10^{10} \mathrm{~N} \cdot \mathrm{s} / \mathrm{m}$, $F=1 \mathrm{MN}$, and $\Delta_{\text {max }}=0.2 \mathrm{~mm}$.

The oversize clearance between mill house and bearing seat can cause roller crossover and roll system transverse vibration; in order to verify the clearance influence on the roll system dynamic characteristics, it is necessary to carry out simulation and analysis. Due to large weight of drum gear spindle, when it has slight eccentric or installation error, which would cause arc tooth meshing impact, the spindle support with arc tooth will play an important role on vibration suppression. But limited by various factors, it is difficult to be implemented at the scene, but these achievements have certain inspiration to rolling mill manufacturers. In order to eliminate the gap and eliminate the phenomenon of roll bearing seat collision on mill house, free expansion hydraulic plate can be used; in order to reduce or eliminate the drum gear shaft imbalance force, the drum gear spindle floating support can be used. In order to test and verify the effect of these measures, the numerical simulation was carried out, respectively, in this paper. In Figure 16, the simulation curve is the roller displacement response diagram, phase diagram, and displacement power spectrum diagram, respectively.

According to the roll horizontal vibration mechanics model (Figure 15), with the gap between roll bearing seat and mill house eliminated, roll horizontal vibration differential equations can be expressed as follows:

$$
m \ddot{x}=-\left(k_{1}+2 k_{2}\right) x+A \dot{x}-B \dot{x}^{3}+F \cos \Omega t \cos 51 \Omega t .
$$
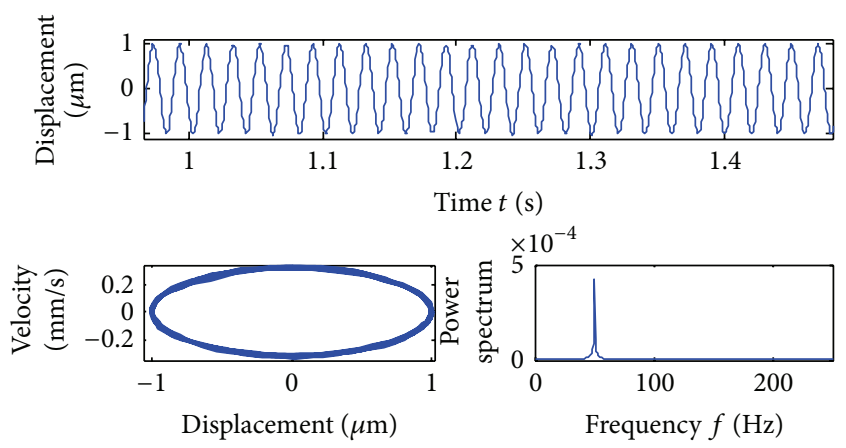

FIGURE 17: F3 horizontal response with measures.

The values of the parameters are the same as the mentioned above. When adding drum spindle floating support, the spindle unbalance excitation disappears; roller horizontal vibration differential equation is expressed as follows:

$$
\begin{aligned}
& m \ddot{x}+f(x, \dot{x})=F \cos 51 \Omega t, \\
& f(x, \dot{x}) \\
& = \begin{cases}-\left(k_{1}+k_{2}\right) x+A \dot{x}-B \dot{x}^{3} & x \geq 0 \text { or } x \leq-\Delta_{\text {max }} \\
-k_{1} x+A \dot{x}-B \dot{x}^{3} & -\Delta_{\text {max }}<x<0 .\end{cases}
\end{aligned}
$$

When the two measures of eliminating the gap between the roller bearing seat and mill house and adding the drum gear shaft floating support were adopted at the same time, roll horizontal vibration differential equations can be expressed as follows:

$$
m \ddot{x}=-\left(k_{1}+2 k_{2}\right) x+A \dot{x}-B \dot{x}^{3}+F \cos 51 \Omega t .
$$

The simulation results are shown in Figure 17 of eliminating the gap between the roller bearing seat and mill house and adding the drum gear shaft floating support, adopted at the same time. From the figure, we can see that after these two measures are taken at the same time, the vibration amplitude reduced to one-tenth of the original, and by the phase diagram and the spectrum diagram, system property has been improved significantly and the roller "jump vibration" phenomenon disappeared.

We adopted vibration suppression measures as adding copper gaskets within the bearing liner to eliminate or reduce 

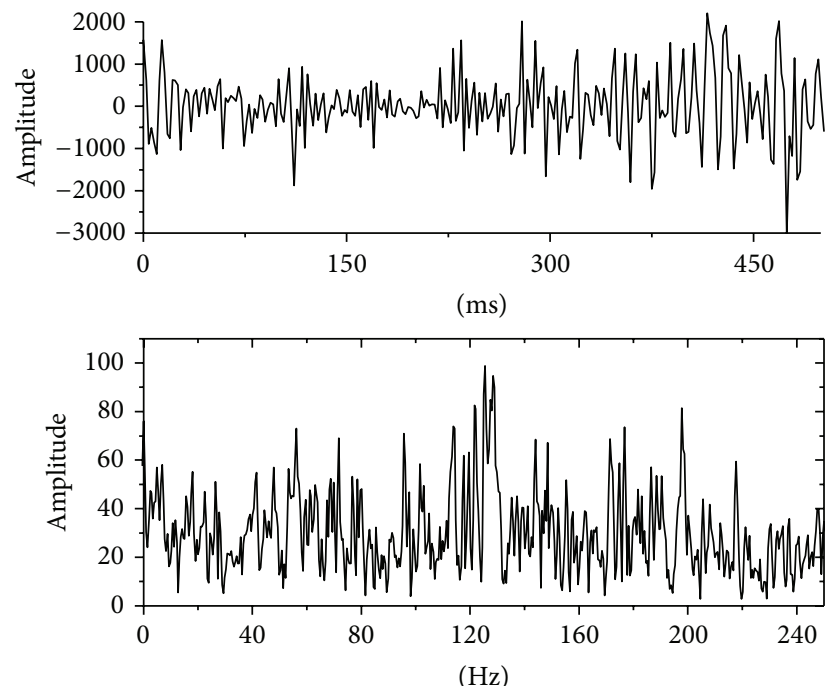

FIGURE 18: Roller horizontal vibration measured results after gap elimination.

the bearing seat and mill house clearance at site. The roller horizontal vibration results after eliminating the clearance are shown in Figure 18, and it can be seen after eliminating the clearance that chopped or touch vibration phenomenon disappears; vibration amplitude is declined greatly and the energy spread. So the measure of gap eliminating can reduce mill vibration (horizontal).

\section{Conclusions}

In order to deeply study the rolling mill vibration source, the roll system structure stability was analyzed and calculated firstly and it had been found that when the F3 rolling mill work roller diameter is small, the offset basically conforms to the requirements of the static stability. Then, the field measurement of the clearance between mill stand and bearing seat has been carried out, and it was found that F3 clearance is much larger than the original design (transition fit) requirement; namely, the gap provides necessary conditions for roller horizontal vibration. The drum gear coupling effect on the roll system stability is analyzed, and it was found that the coupling transmission torque cannot meet self-centering requirement, roller driving system always has unbalanced force or centrifugal force, and this is verified by the field vibration test. At the same time, the axial force by rollers cross caused by roller system structure defect has great destruction on the system stability and it cannot be allowed to be ignored. Finally, the roll horizontal friction chatter vibration mechanics model was established and the simulation analysis was carried out; it showed that when eliminating the gap between the roller bearing seat and mill house and adding the drum gear shaft floating support at the same time, roll vibration amplitude was reduced to one over ten of the original, and by the phase diagram and the spectrum diagram, system dynamics have been improved thoroughly and the roller "jump vibration" phenomenon disappeared. At the same time, adding copper gaskets to eliminate or reduce the clearance between the bearing seat and mill house for vibration suppression at the scene, the results showed that after the clearance is eliminated, chopped or touch vibration phenomenon disappeared and vibration amplitude value has fallen obviously, and the energy has spread; that is, it is helpful to suppress roller horizontal vibration.

\section{Competing Interests}

The authors declare that there are no competing interests regarding the publication of this paper.

\section{Acknowledgments}

This research was supported by National Undergraduate Training Programs for Innovation and Entrepreneurship (no. 201510460001), Henan Polytechnic University Education Teaching Reform Research Projects (no. 2015JG034), and Colleges and Universities Focus on Soft Science Research Project Plan (no. 16A630049).

\section{References}

[1] X. Shu and G. Shen, "Analysis of roll system stability about aluminum foil mills," Chinese Journal of Mechanical Engineering, vol. 16, no. 2, pp. 181-189, 2003.

[2] G. Shen and M. Li, "Statically determinate characteristics of microdisplacement in a four-high mill," Journal of Materials Processing Technology, vol. 209, no. 11, pp. 5002-5007, 2009.

[3] A. Bar and O. Bar, "Types of mid-frequency vibrations appearing during the rolling mill operation," Journal of Materials Processing Technology, vol. 162-163, pp. 461-464, 2005.

[4] A. Bar and A. Świątoniowski, "Interdependence between the rolling speed and non-linear vibrations of the mill system," Journal of Materials Processing Technology, vol. 155-156, no. 11, pp. 2116-2121, 2004.

[5] V. Panjković, R. Gloss, J. Steward, S. Dilks, R. Steward, and G. Fraser, "Causes of chatter in a hot strip mill: observations, qualitative analyses and mathematical modelling," Journal of Materials Processing Technology, vol. 212, no. 4, pp. 954-961, 2012.

[6] H. Furumoto, S. Kanemori, K. Hayashi, and A. Sako, "Enhancing technologies of stabilization of mill vibration by mill stabilizing device in hot rolling," Procedia Engineering, vol. 81, no. 12, pp. 102-107, 2014.

[7] Y. Kim, C.-W. Kim, S.-J. Lee, and H. Park, "Experimental and numerical investigation of the vibration characteristics in a cold rolling mill using multibody dynamics," ISIJ International, vol. 52, no. 11, pp. 2042-2047, 2012.

[8] Y. Kim, H. Park, S. S. Lee, and C.-W. Kim, "Development of a mathematical model for the prediction of vibration in a cold rolling mill including the driving system," ISIJ International, vol. 52, no. 6, pp. 1135-1144, 2012.

[9] A. Swiatoniowski and R. Gregorczyk, "Self-excited vibrations in four-high rolling mills caused by stochastic disturbance of friction conditions on the roll-roll contact surface," Mechanics and Control, vol. 29, no. 3, pp. 158-162, 2010.

[10] Y. A. Amer, A. T. EL-Sayed, and F. T. El-Bahrawy, “Torsional vibration reduction for rolling mill's main drive system via negative velocity feedback under parametric excitation," Journal 
of Mechanical Science and Technology, vol. 29, no. 4, pp. 15811589, 2015.

[11] F. Xiaobin, Vibration problem research for CSP mill stand [Ph.D. thesis], University of Science and Technology Beijing, Beijing, China, 2007. 


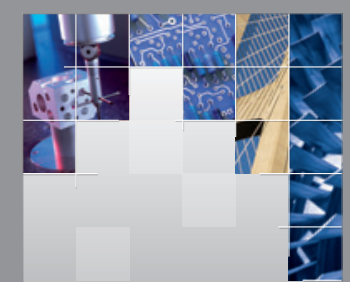

\section{Enfincering}
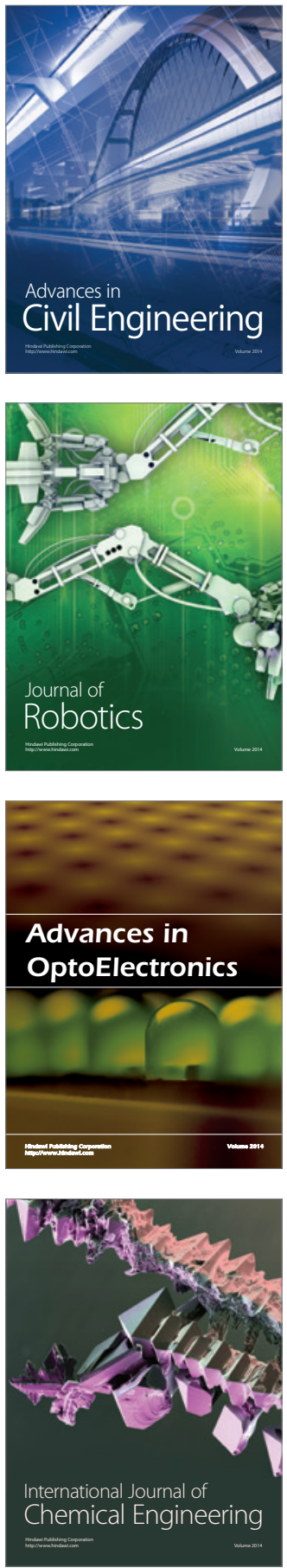

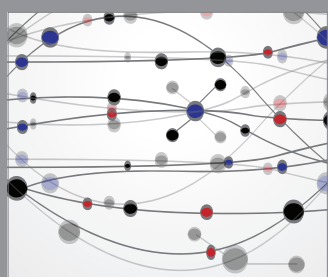

The Scientific World Journal

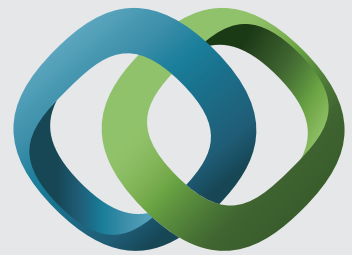

\section{Hindawi}

Submit your manuscripts at

http://www.hindawi.com
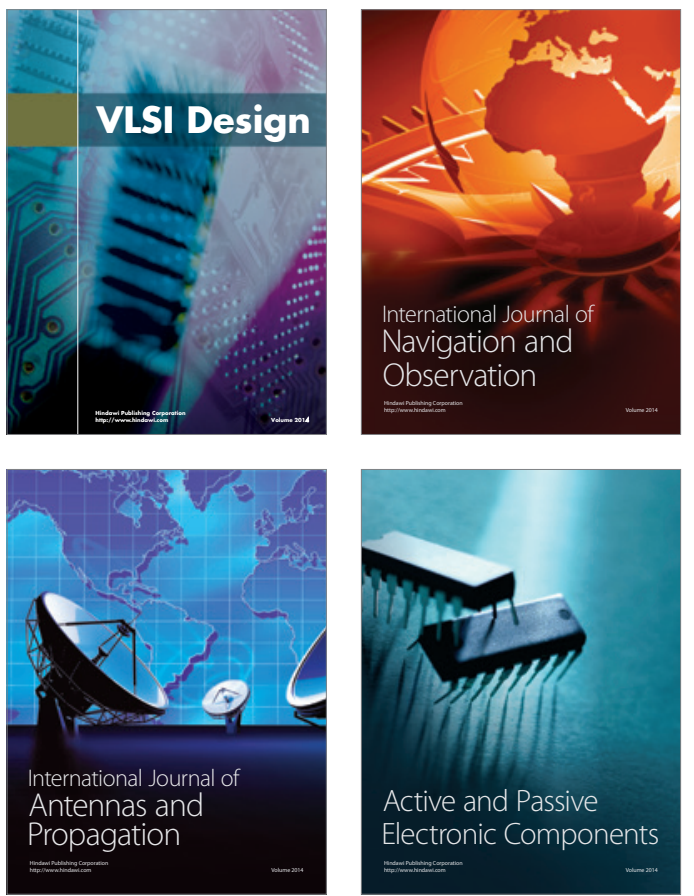
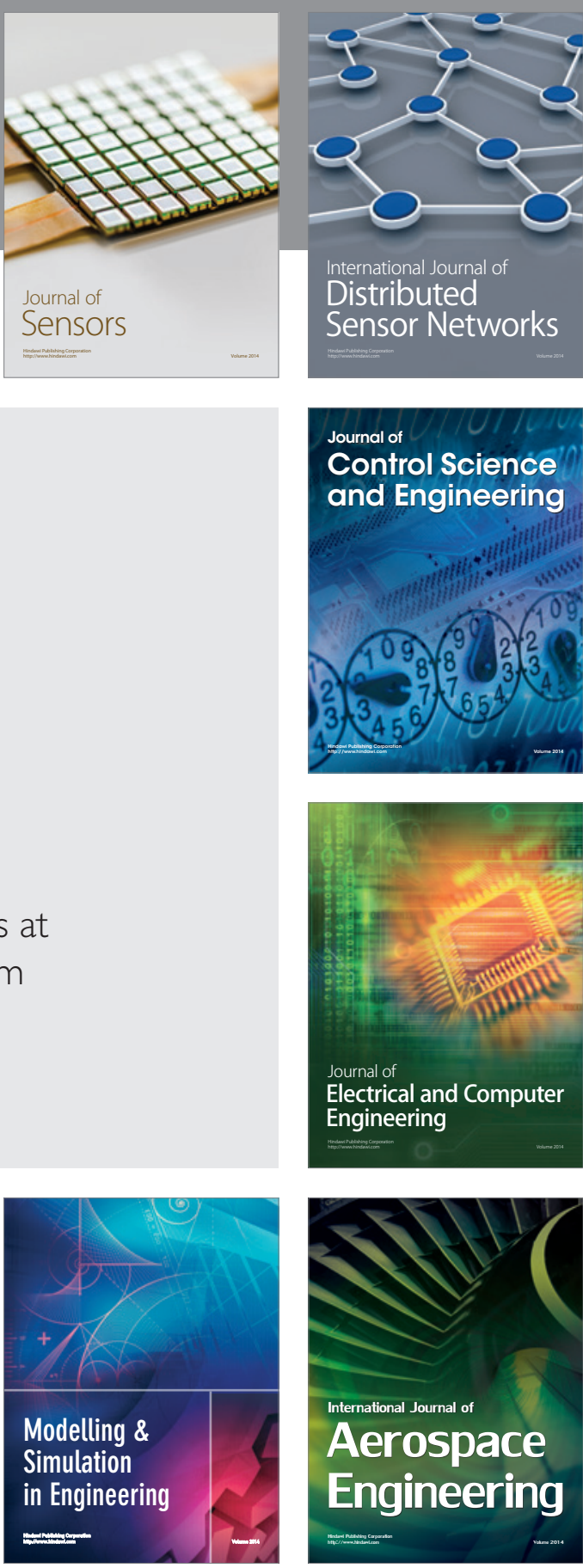

International Journal of

Distributed

Sensor Networks

Journal of

Control Science

and Engineering
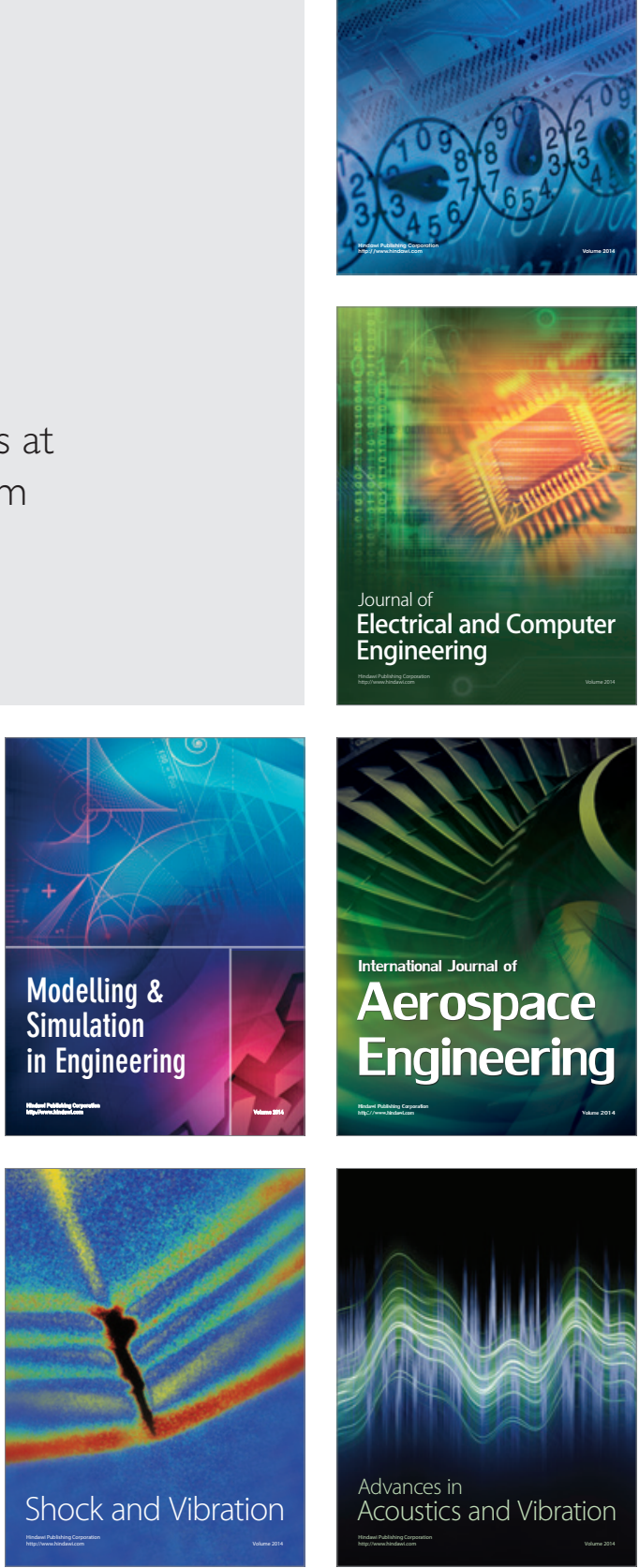